\title{
MEMS Dynamic Characteristics Analysis of Electrostatic Microbeams for Building Structure Monitoring
}

\author{
Youping Gong, Hong Bo, Chuanping Zhou, Maofa Wang, and Rougang Zhou $(\mathbb{D}$ \\ School of Mechanical Engineering, Hangzhou Dianzi University, Hangzhou 310018, China \\ Correspondence should be addressed to Rougang Zhou; zjlhzz@163.com
}

Received 3 October 2021; Revised 25 November 2021; Accepted 11 December 2021; Published 19 January 2022

Academic Editor: Lingkun Chen

Copyright (c) 2022 Youping Gong et al. This is an open access article distributed under the Creative Commons Attribution License, which permits unrestricted use, distribution, and reproduction in any medium, provided the original work is properly cited.

\begin{abstract}
Building structure health monitoring is essential for modern buildings, sensors related to building structure health monitoring are often made with microelectrostatic cantilever beam (MECB), and the performance of this kind of devices is often affected by instability, which affects the measurement results and accuracy. Therefore, it is necessary to study the nonlinear dynamic characteristics of the MECB in the process of bending and pull-in. In this paper, based on the energy principle and fluid pressure film damping effect, the dynamic equation mathematical model of MECB is established and then the dynamic characteristics of the pull-in and lift-off voltage of the MECB and the harmonic motion characteristics under the bias voltage are obtained, which provides guidance for the design of the electrostatic driving sensor.
\end{abstract}

\section{Introduction}

Building structure health monitoring is essential for modern buildings. Due to the action of various external forces (i.e., loads), building structures will produce certain damage. Therefore, a method that can monitor the structural health for a long time and make safety early warning is needed [1-4]. Sensors related to building structure health monitoring technology have become a research hotspot. Electrostaticdriven multilayer cantilever beams are widely used in building structure monitoring sensors. Fatigue effect, material aging, accidental load, and other factors have great influence on the structure. In recent years, there are numerous examples of loss of life and properties caused by structural instability $[5,6]$. This makes the building structure health monitoring become an urgent need. The research of building structure monitoring systems based on MEMS has become a cutting-edge topic in the field of civil engineering. As the basic structural devices of many MEMS devices, microelectrostatic cantilever beams (MECBs) are widely used in RF switches, low-light switches, and pressure sensors, as well as MEMS devices in building structure monitoring systems [7-10]. However, due to the inherent instability of electromechanical coupling, that is, the so-called pull-in phenomenon, the performance of these devices is often affected by instability. In addition, due to the multicoupling and nonlinear characteristics of the MECB cantilever beam, the multiparameter simulation design method has the disadvantages of high cost, slow speed, and insufficient accuracy. Therefore, in the process of device-level design of building monitoring MEMS, it is increasingly important to find a high-precision, high-economic and fast optimization method for MECB.

At present, the most accurate method for solving the device-level dynamic characteristics of MEMS is to establish an effective partial differential equation (PDES) to describe the nonlinear dynamic characteristics of the system in mathematical form through the analysis of its working principle and coupled physical model and then solve it by finite element method (FEM), boundary element method (BEM), finite difference method (FDM), and other methods after selecting effective control parameters. To improve the accuracy of the calculation, the model needs to be divided into a very detailed grid to solve, the refinement of the grid will inevitably lead to the allocation of a large number of resources, which cannot meet the solution under multiparameters and multiexcitation, so the optimization efficiency of the parameter design space is very low, and it is even more difficult to deal with the MEMS devices of multidevices 
[11-16]. In this paper, according to the principle of conservation of energy, the MECB model of the building monitoring MEMS is constructed. The model is solved in Simulink, and the dynamic characteristics of the MECB under pull-in and lift-off voltage and the harmonic motion characteristics under bias voltage are obtained. The research results of this paper will greatly improve the development and application of building monitoring MEMS system.

\section{Construction of Dynamic Characteristic Model of MECB Based on Energy Method}

As shown in Figure 1, the MEMS electrostatic microbeam's length is $l$, width is $w$, and thickness is $h$, and the $x$ axis is established on the neutral axis of the beam, and the $y$ axis is located at the left end of the beam. In this case, it is assumed that the material of the microbeam is homogeneous, Young's modulus is $E$, and Poisson's ratio is $\delta$. The initial in-plane load of the beam is affected by $N_{0 x}$ or residual stress $s$.

Then, the relationship between stress and strain of the microbeam can be expressed by the following equation:

$$
\sigma_{x}=\frac{E}{\left(1-\delta^{2}\right)} \varepsilon_{x}+\frac{1}{w h} N_{o x} .
$$

In formula (1), $E$ is the equivalent modulus, and axial strain force of the beam can be expressed as the following equation:

$$
\varepsilon_{x}=\frac{\partial \widehat{u}}{\partial \widehat{x}}-\frac{\partial^{2} \widehat{y}}{\partial^{2} \widehat{x}} \widehat{y}+\frac{1}{2}\left(\frac{\partial \widehat{y}}{\partial \widehat{x}}\right)^{2} .
$$

The elastic deformation energy in the beam volume can be expressed as the following equation:

$$
\begin{aligned}
U_{s t}= & \frac{1}{2} \iiint_{V} \varepsilon_{x} \sigma_{x} \mathrm{~d} V=\frac{E A}{2} \int_{0}^{l}\left(\frac{\partial \widehat{u}}{\partial \widehat{x}}\right)^{2} \mathrm{~d} \widehat{x} \\
& +\frac{E I}{2} \int_{0}^{l}\left(\frac{\partial^{2} \widehat{y}}{\partial \widehat{x}}\right)^{2} \mathrm{~d} \widehat{x}+\frac{E A}{2} \int_{0}^{l}\left(\frac{\partial \widehat{u}}{\partial \widehat{x}}\right)\left(\frac{\partial^{2} \widehat{y}}{\partial \widehat{x}}\right)^{2} \mathrm{~d} \widehat{x} \\
& +\frac{E A}{2} \int_{0}^{l} \sigma_{0}\left(\frac{\partial \widehat{u}}{\partial \widehat{x}}\right) \mathrm{d} \widehat{x}+\frac{E A}{4} \int_{0}^{l} \sigma_{0}\left(\frac{\partial \widehat{y}}{\partial \widehat{x}}\right)^{2} \mathrm{~d} \widehat{x} .
\end{aligned}
$$

In equation (3), $I=w h^{3} / 12$, which is moment of inertia, $\rho$ is the density of material, and the third term is the geometric nonlinear coupling term due to large deformation. The axial force on the neutral plane can be expressed as $\partial \widehat{u} / \partial \widehat{x}=1 / 2 l \int_{0}^{l}(\partial \hat{y} / \partial \widehat{x})^{2} \mathrm{~d} \hat{x}$. Equation (4) can be obtained by substituting it into equation (3):

$$
U_{s t}=\frac{E I}{2} \int_{0}^{l}\left(\frac{\partial^{2} \hat{y}}{\partial \hat{x}}\right)^{2} \mathrm{~d} \hat{x}+\frac{1}{2} N_{o x} \int_{0}^{l}\left(\frac{\partial^{2} \widehat{y}}{\partial \hat{x}}\right)^{2}+\frac{E w h}{8 l}\left[\left(\frac{\partial^{2} \hat{y}}{\partial \hat{x}}\right)^{2} \mathrm{~d} \hat{x}\right]^{2}
$$

2.1. MECB Energy Equation. The electrostatic common energy of the above system can be expressed as the following equation:

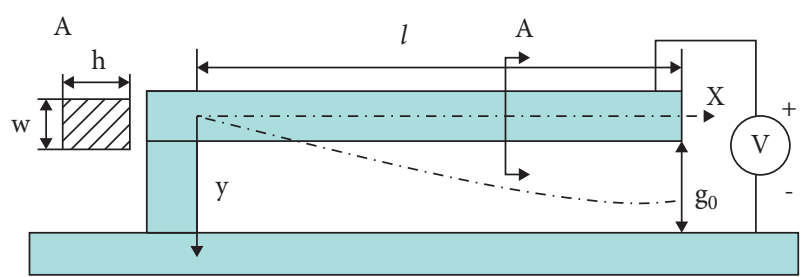

FIgURE 1: Structural diagram of microcantilever.

$$
U_{E}=-\frac{\varepsilon w V^{2}}{2} \int_{0}^{l} \frac{1}{\left(g_{0}-\hat{y}\right)} \mathrm{d} \widehat{x} .
$$

In equation (5), the formula of parallel plate capacitor is adopted. At this time, the edge effect of the electrostatic field is not considered. In some cases, the influence of edge effect needs to be considered. The correction formula is as follows:

$$
U_{E}=-\frac{\varepsilon w V^{2}}{2} \int_{0}^{l} \frac{1}{\left(g_{0}-\hat{y}\right)}\left[1+\frac{\left(g_{0} / l\right)^{2}}{3} \cdot \frac{1}{g_{0}^{2}}\left(\frac{\partial}{\partial x}\left(g_{0}-\hat{y}\right)\right)^{2}\right] \mathrm{d} \hat{x} .
$$

2.2. Deformation Equation of $M E C B$. The deformation equation of the beam can be derived by using the Lagrangian equation as follows:

$$
E I \frac{\partial^{4} \hat{y}}{\partial^{4} \hat{x}}-\left[N_{o x}+\frac{E w h}{2 l} \int_{0}^{l}\left(\frac{\partial \hat{y}}{\partial \hat{x}}\right) d \widehat{x}\right] \frac{\partial^{2} \hat{y}}{\partial^{2} x}=f_{\text {elec }}(\hat{x}, t \hat{t})-w h \rho \frac{\partial^{2} \hat{y}}{\partial^{2} \widehat{t}},
$$

where $f_{\text {elec }}(x, t)$ is the expression of electrostatic load on the parallel plate beam as follows:

$$
f_{\text {elec }}(x, t)=\frac{1}{2} \frac{\varepsilon w V^{2}}{\left(g_{0}-\widehat{y}\right)} .
$$

According to the energy principle of the system, the equation describing its dynamics can be obtained as the following equation:

$$
\begin{aligned}
E I \frac{\partial^{4} \widehat{y}}{\partial^{4} \hat{x}} & -\left[N_{o x}+\frac{E w h}{2 l} \int_{0}^{l}\left(\frac{\partial \widehat{y}}{\partial \widehat{x}}\right) \mathrm{d} \widehat{x}\right] \frac{\partial^{2} \widehat{y}}{\partial^{2} x}=f_{\text {elec }}(\widehat{x}, \widehat{t}) \\
& +f_{\text {air }}(\hat{x}, y, t)-w h \rho \frac{\partial^{2} \widehat{y}}{\partial^{2} \widehat{t}} .
\end{aligned}
$$

The dimensionless displacement of the beam can be expressed by the product expansion of the spatial and timedependent modal coordinates and the global function $\left\{\phi_{k}(x)\right\}$ as the following equation:

$$
y(x, t)=\sum_{i=1}^{\mathrm{Mv}} q_{i}(t) \phi_{i}(x) .
$$

In equation (10), basis function $\left\{\phi_{i}(x)\right\}$ can be expressed as the set of functions satisfying any boundary condition. Here, $\mathrm{Mv}$ is the number of basis functions. Through the transformation of the above formula, the displacement of the beam in the original physical coordinate system can be 
transformed into the expression in the generalized state $q_{i}(t)$. The number of modes $\mathrm{Mv}$ is usually much smaller than the number of dimensions obtained by the common grid method, so the reduced order model can be obtained, and the accuracy can be controlled by selecting the number of modes. Through this method, the calculation consumption of the model can be reduced and the calculation speed can be improved. The dimensionless displacement $y$ of the beam can be expressed in the product expansion form of spatial and time-dependent modal coordinates and global function.

Substituting formula (9) into (10) and making the residual as zero, then the following expression can be obtained:

$$
\begin{aligned}
\sum_{i=1}^{\mathrm{Mv}} q_{i}(t) \int_{0}^{1} \phi_{j} \phi_{i} \mathrm{~d} x+\sum_{i=1}^{\mathrm{Mv}} q_{i}(t) \int_{0}^{1} \phi_{j}^{\prime \prime} \phi_{i}^{\prime \prime} \mathrm{d} x+N_{0} \sum_{i=1}^{M v} q_{i}(t) \\
\cdot \int_{0}^{1} \phi_{j}^{\prime} \phi_{i}^{\prime} \mathrm{d} x+\alpha_{1} \sum_{m, n, i=1}^{\mathrm{Mv}} q_{m}(t) q_{n}(t) q_{i}(t) \\
\cdot \int_{0}^{l} \phi_{m}^{\prime} \phi_{n}^{\prime} \mathrm{d} x \int_{0}^{1} \phi_{m}^{\prime} \phi_{n}^{\prime} \mathrm{d} x=\alpha_{2} V^{2} \\
\cdot \int_{0}^{1} \frac{\phi_{j}}{\left(1-\sum_{i=1}^{M v} q_{i}(t) \phi_{i}\right)} \mathrm{d} x,(j=1,2, \ldots, \mathrm{Mv}) .
\end{aligned}
$$

In equation (11), the third term is related to the axial tension, and the electrostatic force load term is on the right of the formula. It can be obtained by transforming it into matrix form as follows:

$$
M \ddot{q}+\left(K+N_{0} S\right) q+\alpha_{1} q^{T} S q S q+f=0 .
$$

In equation (12), $M=\left[M_{i j}\right]$ is mass matrix, $K=\left[K_{i j}\right]$ is bending stiffness matrix, and $N_{0} S=N_{0}\left[S_{i j}\right]$ is the stiffness matrix resulting from the initial axial load. Now, consider the solution result of the model in the case of single degree of freedom and make $\mathrm{Mv}=1$; here, $y(x, t)=g(t) \phi_{1}(x)$; from (11), we can obtain the following equation:

$$
\begin{aligned}
\ddot{g} \int_{0}^{1} \phi_{1}^{2} \mathrm{~d} x & +g\left(\int_{0}^{1}\left(\phi_{1}^{\prime \prime}\right)^{2} \mathrm{~d} x+N_{0} \int_{0}^{1}\left(\phi_{1}^{\prime}\right)^{2} \mathrm{~d} x\right) \\
& +g^{3} \alpha_{1}\left(\int_{0}^{1}\left(\phi_{1}^{\prime}\right)^{2} \mathrm{~d} x\right)^{2} \\
& =V^{2} \alpha_{2} \int_{0}^{1}\left(\frac{\phi_{1}}{\left(1-g \phi_{1}\right)}\right) \mathrm{d} x .
\end{aligned}
$$

The simulation model of the MECB is established according to formula (13), and it can be dynamically simulated and solved, as shown in Figure 2.

2.3. Study on the Dynamic Process of MECB Pull-In and Lift-Off. The pull-in and lift-off process of the MECB is studied. The detailed parameters are listed in Table 1.

By adding a triangular wave voltage described in the simulation model established in the above process and taking the damping coefficient as $B=2$, the relationship between the end point of the micro cantilever and the voltage can be obtained, as shown in Figure 3. With the linear increase in the input voltage, the end displacement of the microcantilever does increase nonlinearly. When the pull-in voltage is exceeded, the beam end point suddenly accelerates and pulls down and pulls in to the substrate, resulting in the pull-in voltage. With the decrease in the voltage, when it falls below the pull-in voltage, it will not be separated immediately, because the electrode spacing after the pull-in is very small and the electrostatic load is very large, so it will not separate immediately even if it drops below the pull-in voltage. It can be observed from the figure that when the voltage drops to about $3.2 \mathrm{~V}$, the deformation recovery force of the beam is greater than the electrostatic attraction, and the lift-off phenomenon occurs at this time. It can be found that the liftoff voltage is much smaller than the pull-in voltage and has a lag phenomenon, which is of great significance for the research of sensors and actuators working with pull-in voltage.

The pull-in the curve can be obtained from the singlemode model established by Simulink, as shown in Figure 4; it can be observed that the pull-in voltage is about $8.75 \mathrm{v}$.

The working mode of microresonance is to apply DC voltage and alternating driving voltage to the upper plate of the fixed microbeam. The DC voltage can make it reach an equilibrium position, and the alternating current can make it simple harmonic vibration up and down at this equilibrium position. In this process, the equilibrium state is curved, and there is a gap between the modal vibration mode and the straight beam. At this time, using the modal vibration mode of the straight beam as the basis function will have the possibility of error, resulting in the inaccuracy of the calculated dynamic characteristics [17]. Therefore, to verify that the reduced order model has sufficient accuracy to simulate and calculate the dynamic characteristics of MEMS microbeam under resonant voltage, at this time, the dynamic characteristics of the device model based on the above data are simulated and calculated when the input voltage signal of the device is $\mathrm{v}$. The steady-state response diagram of the end displacement of the beam calculated by the reduced order model is shown in Figure 5. To verify the correctness of the above model verification, the same simulation is done in ConventorWare, and the result is shown in Figure 6.

From Figure 5, it can be seen that the equilibrium center point of the results calculated by the simulation model is $-0.647 \mu \mathrm{m}$, the upper limit change displacement after stabilization is $-0.748 \mu \mathrm{m}$, and the lower limit displacement is $-0.546 \mu \mathrm{m}$, while the analysis results inConventorWare show that the equilibrium point position is stable at $-0.643 \mu \mathrm{m}$, the upper limit displacement after stabilization is $-0.748 \mu \mathrm{m}$, and the lower limit is $-0.565 \mu \mathrm{m}$, as shown in Figure 6. The results show that the two analysis results are consistent which verifies the effectiveness of our proposed model and can calculate the dynamic characteristics of the beam under bias voltage. We established the physical simulation model of the electrostatic microbeam in ConventorWare software. The specific parameters are shown in the simulation model. Voltage is applied continuously to it to obtain its lift-off voltage diagram. The results are shown in Figure 7. 


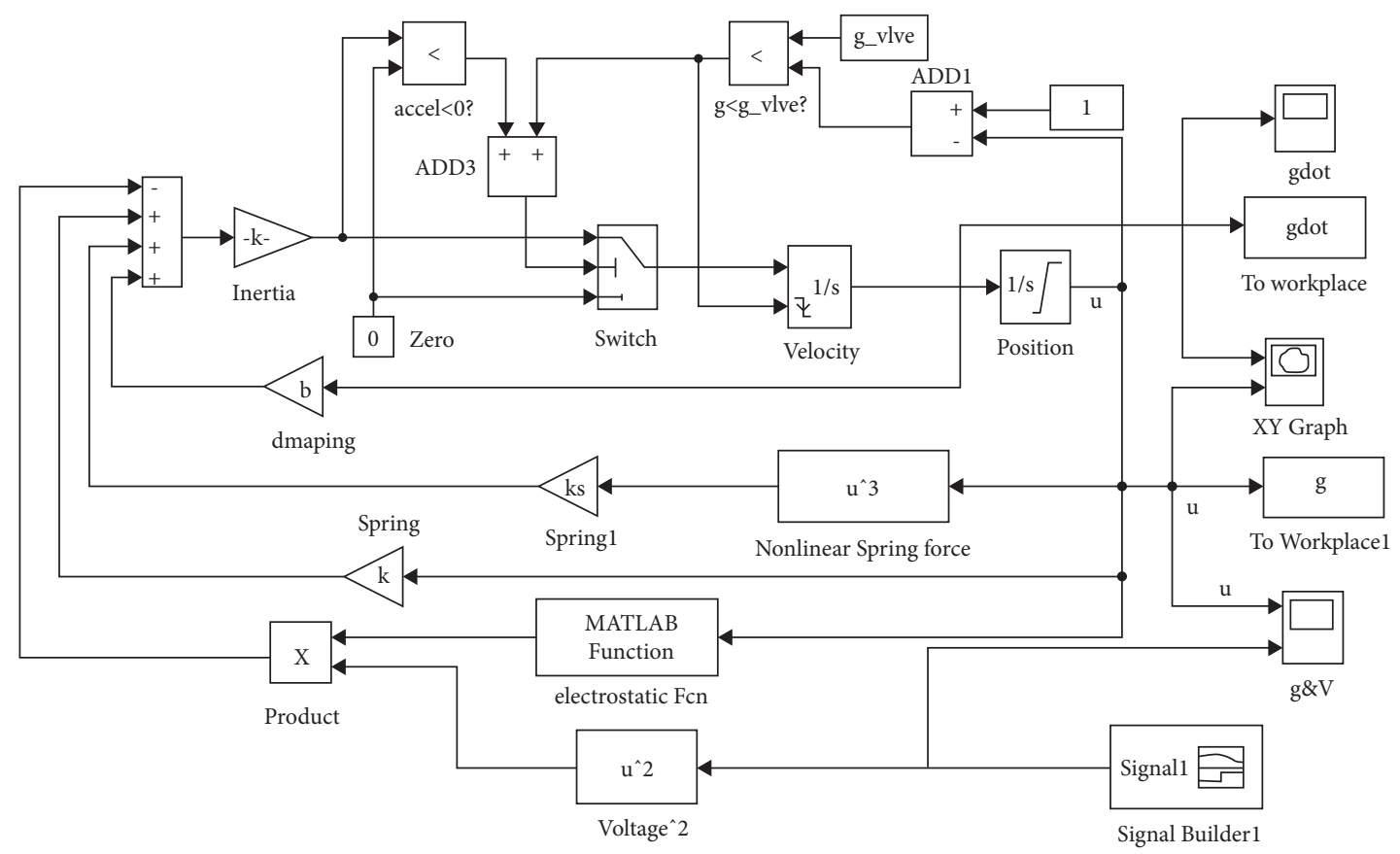

FIgURE 2: The simulation model of the MECB device.

TABLe 1: Multilayer microbeam parameters.

\begin{tabular}{lcc}
\hline Type & Symbol & Data \\
\hline Length & $L$ & $600 \mu \mathrm{m}$ \\
Width & $w$ & $40 \mu \mathrm{m}$ \\
Thickness & $H$ & $2.4 \mu \mathrm{m}$ \\
Young modulus & $E$ & $150 \mathrm{GPa}$ \\
Air viscosity & $U$ & $1.82^{*} 10-5 \mathrm{~kg} /\left(\mathrm{m}^{*} \mathrm{~s}\right)$ \\
Residual stress & $S$ & $-3.7 \mathrm{MPa}$ \\
Density & $V(t)=8+0.3 \sin (2 \pi f) \mathrm{V}$ & $2330 \mathrm{~kg} / \mathrm{m}^{3}$ \\
Air pressure & $\mathrm{P} 0$ & $1.013 * 105 \mathrm{pa}$ \\
\hline
\end{tabular}

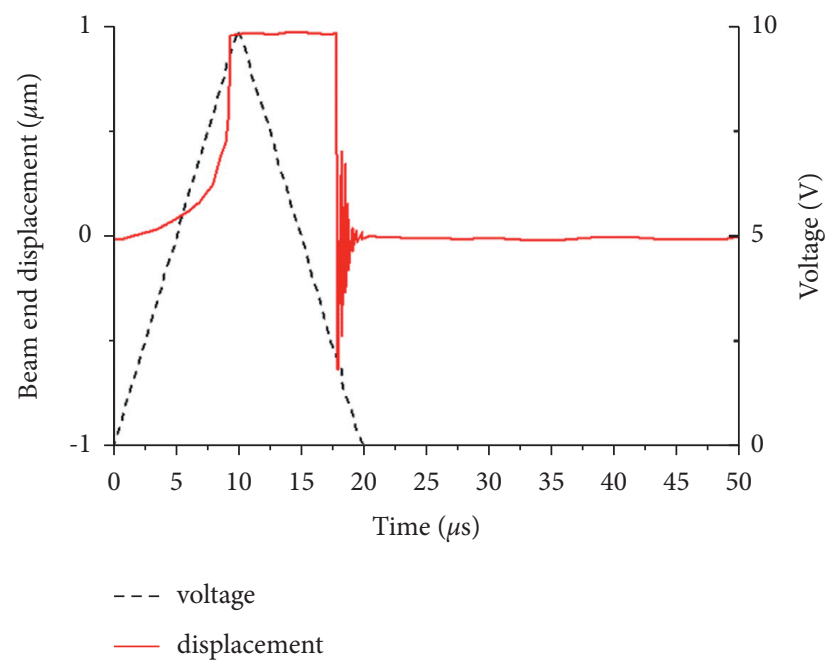

Figure 3: Variation diagram of voltage and displacement from pull-in to lift-off.

From Figure 7, the lift-off voltage is $3.1875 \mathrm{v}-3.375 \mathrm{v}$, which is consistent with the result of $3.2 \mathrm{~V}$ obtained in the price reduction process of the above model. We can also learn that before the pull-in phenomenon, the deformation of MEMS microcantilever is relatively small and the deformation force is relatively small. At this time, it can be observed in Figure 7 that 


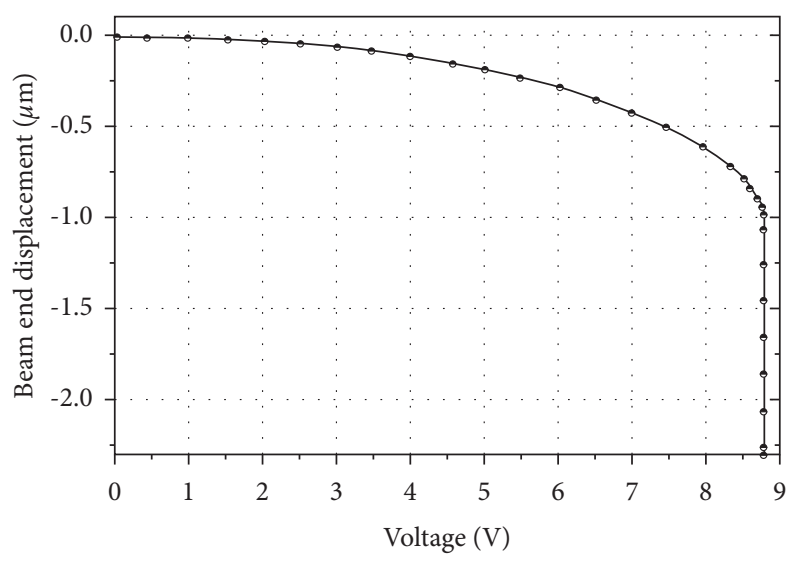

Figure 4: Pull-in process.

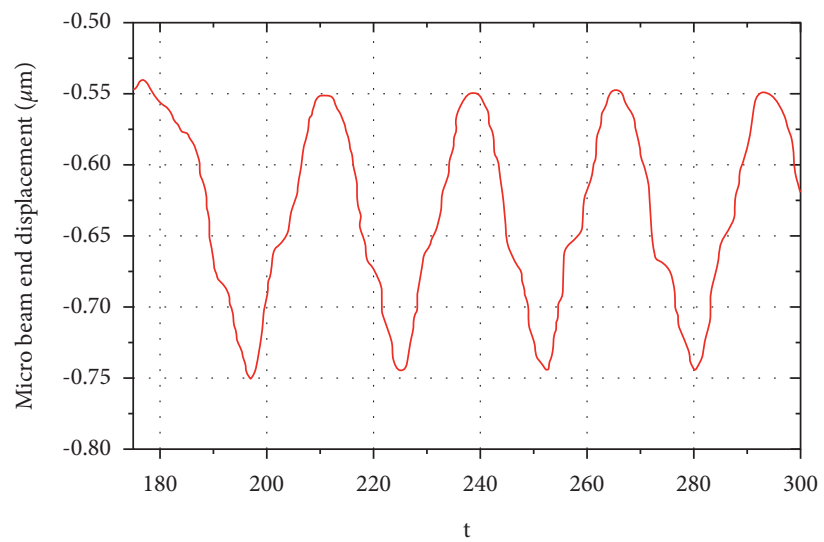

FIGURE 5: Steady-state response diagram of resonant motion under the action of bias voltage and superimposed sinusoidal voltage.

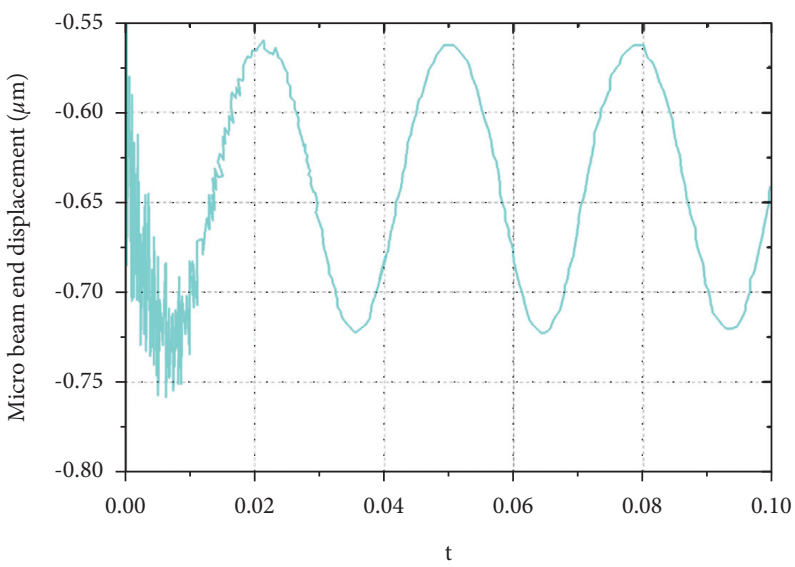

FIgURE 6: Steady-state response curve under ConventorWare analysis.

the linear spring restoring force is much larger than the nonlinear restoring force, which plays a leading role. With the increase in voltage, the nonlinear spring restoring force increases sharply; at this time, the nonlinear spring restoring force and linear restoring force act on the microcantilever at the same time. At this time, if the research on the nonlinear spring restoring force is ignored, it will have a great impact on the liftoff voltage. It is verified again that the microcantilever has very strong nonlinear characteristics under the coupling field. Therefore, the large deformation geometric nonlinearity must be considered in the research of pull-in process.

2.4. Study on the Instability of MECB. The quasi-static pull-in and lift-off characteristic analysis of the MECB cannot accurately express the stability of the microcantilever at the equilibrium position. Under the working conditions, the 
$6 \mathrm{v}$
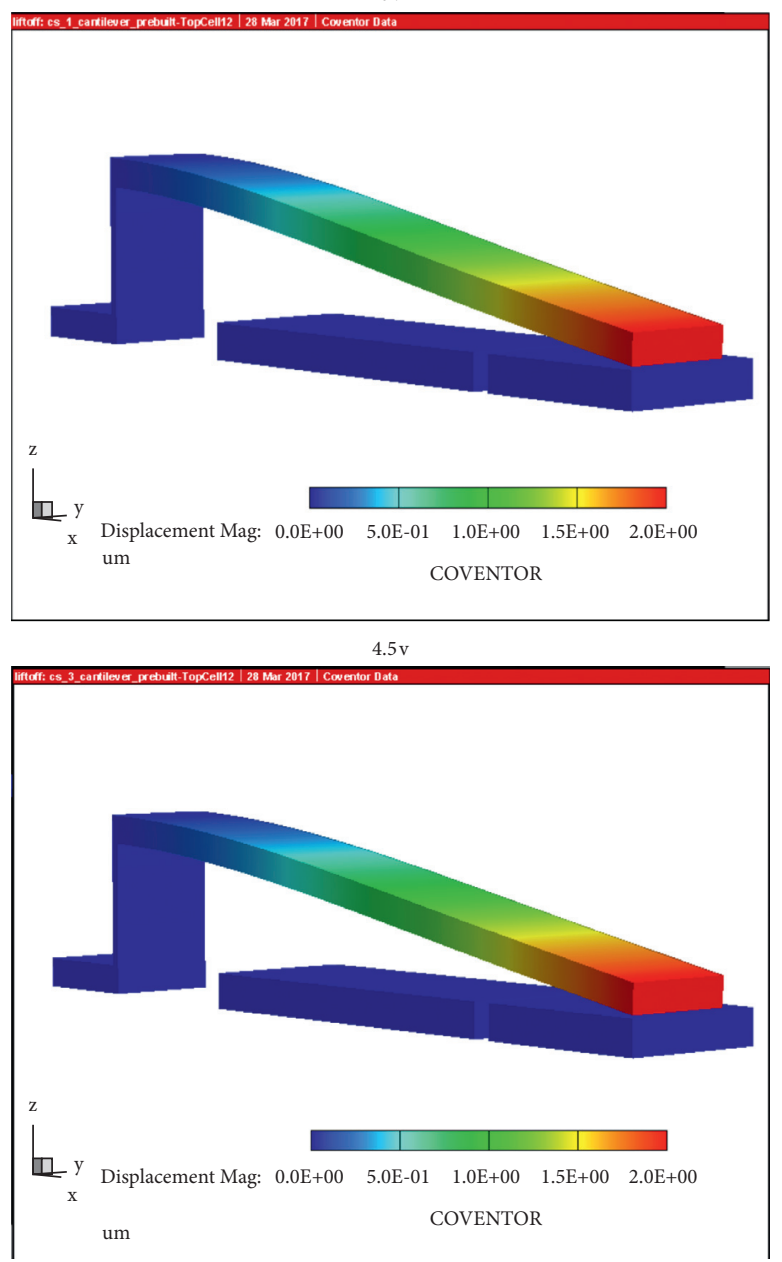

$3.375 \mathrm{v}$

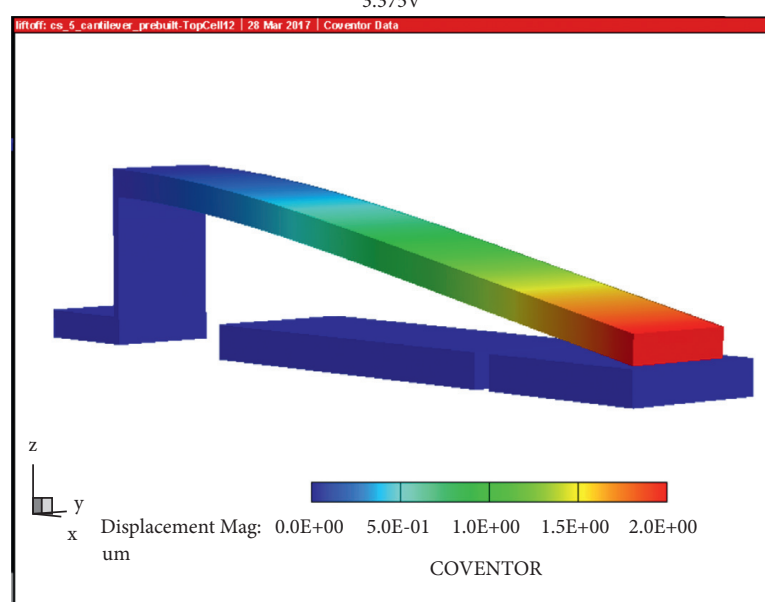

$3 \mathrm{v}$
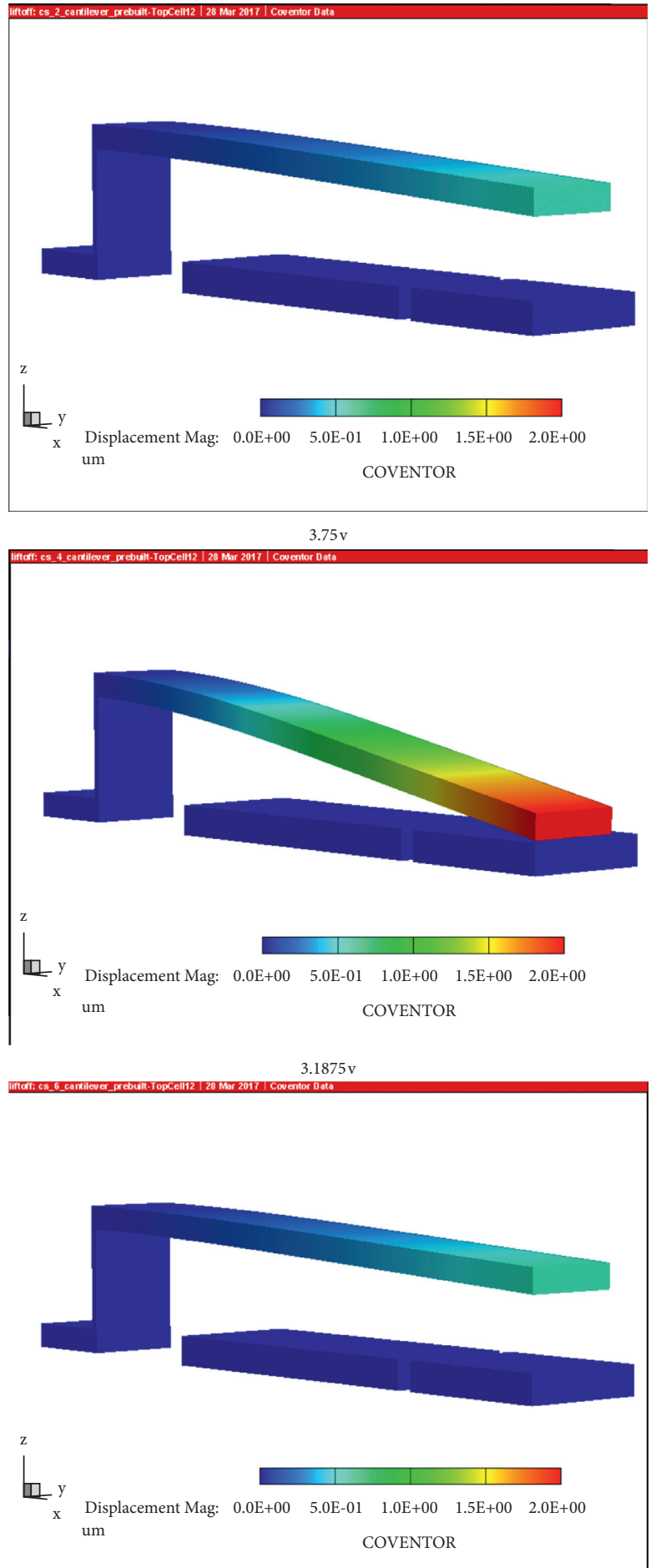

Figure 7: Iterative solution of lift-off voltage in ConventorWare: (a) $6 \mathrm{v}$; (b) $3 \mathrm{v}$; (c) $4.5 \mathrm{v}$; (d) $3.75 \mathrm{v}$; (e) $3.375 \mathrm{v}$; (f) $3.1875 \mathrm{v}$.

instability of the electrostatic microbeam will be caused by the action of step voltage and inertial force in advance. The stable position of the microbeam can be obtained by solving the changes of the dynamic characteristics of the microbeam under different step voltages in the initial state [18, 19]. Figure $8(a)$ shows the change history of microbeam end displacement with time under different step voltages without damping, and Figure 8(b) is its corresponding phase plan. It can be observed from the figure that there is an overshoot phenomenon in the microbeam under the step voltage; that is, the pull-in phenomenon also occurs when the pull-in voltage calculated by the steady state has not been reached. The pull-in voltage at this time is called the dynamic pull-in voltage. It can be observed that the calculated voltage is about $8.1 \mathrm{v}$, which is only $91.3 \%$ of the static voltage at this time. When it is lower than the dynamic pull-in voltage, 


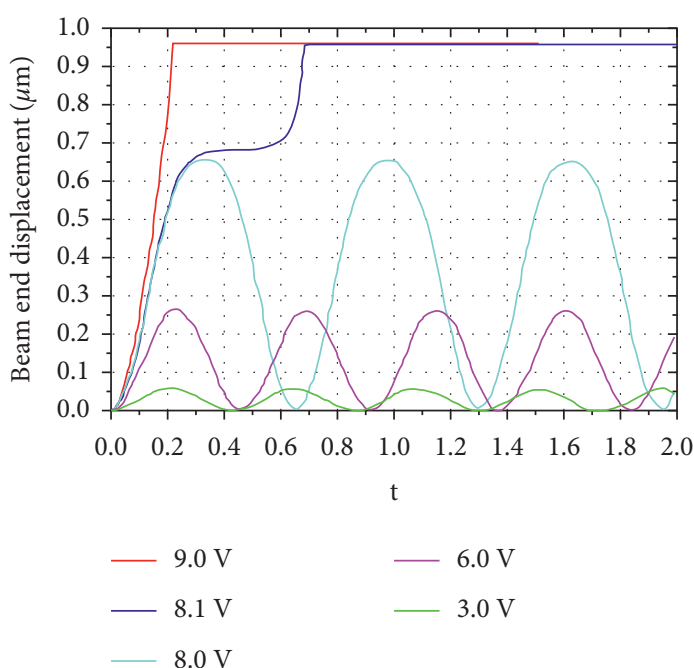

(a)
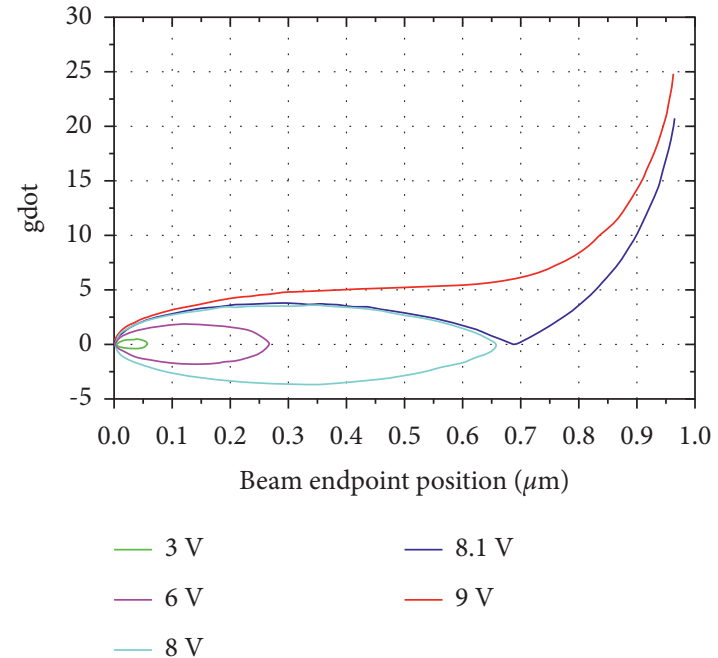

(b)

FIGURE 8: Different step voltages without damping: (a) time history diagram; (b) phase plan.

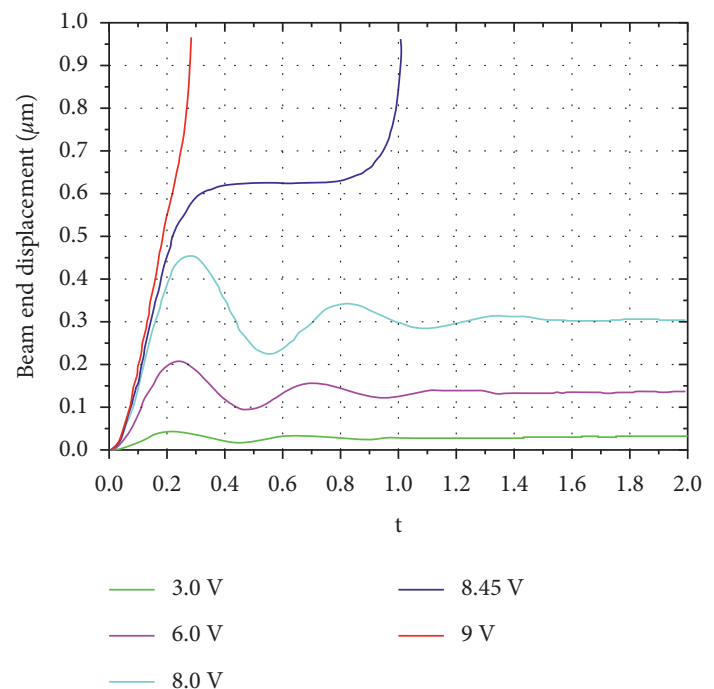

(a)

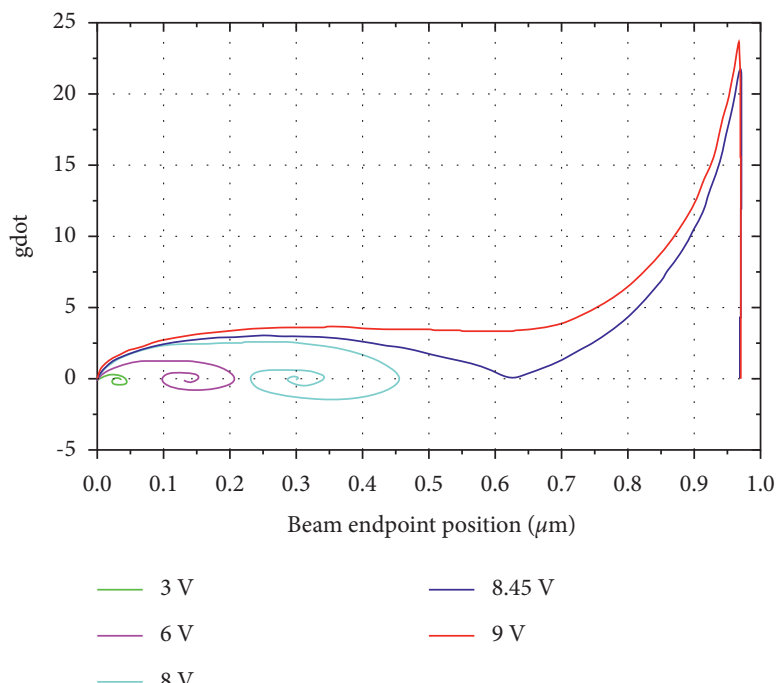

(b)

Figure 9: Different step voltages with damping: (a) time history diagram; (b) phase plan.

because it is set to be undamped at this time, the microbeam makes a fixed simple harmonic vibration at a certain frequency at the equilibrium position. It can be observed in the figure that as the voltage increases, the amplitude increases, but its vibration frequency decreases. When the input voltage is greater than the dynamic pull-in voltage, there is a bifurcation phenomenon in the phase plan, which indicates a pull-in phenomenon.

Figures 9(a) and 9(b) show the displacement diagram and phase plan of the end point of the microbeam with different step voltages under the condition of damping. It can be observed from the figure that $V_{\mathrm{DPI}}$ is about $8.45 \mathrm{v}$ with damping, which is different from that without damping but lower than the static pull-in voltage. It can be seen that the damping will also affect the dynamic pull-in voltage. When it is lower than the microbeam $V_{\mathrm{DPI}}$ at an equilibrium position, the attenuation motion is made. When it is higher than the microbeam $V_{\mathrm{DPI}}$, the phase plan bifurcation occurs. At this time, the microbeam is pulled down quickly. When the voltage is close to the critical point, the small voltage change will affect the response of MEMS microbeam. Therefore, the research on the pull-in voltage of MEMS is of great significance, and the dynamic pull-in voltage stability of microbeam has a certain relationship with damping, which should be further studied.

\section{Conclusion}

In this paper, a method for calculating the MECB model is proposed. The main parameters of the beam model are equivalent to those of the single-layer cantilever beam, and then the dynamic model of the electrostatic microbeam was 
constructed. Aiming at the characteristics of multifield coupling and strong nonlinearity in the process of solving microbeam, the solution method is proposed to express the solution system as a set of global orthogonal spatial basis functions with finite number, so that the simulation process of the dynamic model of microbeam structure can be realized with certain accuracy, fast solution.

\section{Data Availability}

The data used to support the findings of this study are included within the article.

\section{Conflicts of Interest}

The authors declare that they have no conflicts of interest.

\section{Acknowledgments}

The work was financially supported by the National Natural Science Foundation of China (51805475, 51475129, 51275141, and 51305113), Key Discipline of the Ocean Techtronic Equipment's Technology, Nature Science Foundation of Zhejiang Provinces Technology (51475129), and 3D Assembly of Complex Biological Structure (LY14E050026).

\section{References}

[1] A. Norris, M. Saafi, and P. Romine, "Temperature and moisture monitoring in concrete structures using embedded nanotechnology/microelectromechanical systems (MEMS) sensors," Construction and Building Materials, vol. 22, no. 2, pp. 111-120, 2008.

[2] K. Hong-Jin, "System identification of a building structure using wireless MEMS system," Transactions of the Korean Society for Noise and Vibration Engineering, vol. 18, no. 4, pp. 458-464, 2008.

[3] P. Ragam and N. Devidas Sahebraoji, "Application of MEMSbased accelerometer wireless sensor systems for monitoring of blast-induced ground vibration and structural health: a review," IET Wireless Sensor Systems, vol. 9, no. 3, pp. 103-109, 2019.

[4] Č. Kristýna, V. Lukáš, V. . Jan, D. Milan, and Š. Ladislav, "Environmental testing of a FBG sensor system for structural health monitoring of building and transport structures," Procedia Structural Integrity, vol. 17, pp. 726-733, 2019.

[5] G. J. Yun, S.-G. Lee, J. Carletta, and T. Nagayama, "Decentralized damage identification using wavelet signal analysis embedded on wireless smart sensors," Engineering Structures, vol. 33, no. 7, pp. 2162-2172, 2011.

[6] T. Torfs, T. Sterken, S. Brebels et al., "Low power wireless sensor network for building monitoring," IEEE Sensors Journal, vol. 13, no. 3, pp. 909-915, 2013.

[7] M. Moghimi Zand and M. T. Ahmadian, "Application of homotopy analysis method in studying dynamic pull-in instability of microsystems," Mechanics Research Communications, vol. 36, no. 7, pp. 851-858, 2009.

[8] G. De Pasquale and A. Somà, "Dynamic identification of electrostatically actuated MEMS in the frequency domain," Mechanical Systems and Signal Processing, vol. 24, no. 6, pp. 1621-1633, 2010.
[9] B. Krijnen and D. M. Brouwer, "Flexures for large stroke electrostatic actuation in MEMS," Journal of Micromechanics and Microengineering, vol. 24, p. 1, Article ID 015006, 2013.

[10] G. C. Brown and R. J. Pryputniewicz, "Experimental and computational determination of dynamic characteristics of microbeam sensors," Proceedings of SPIE - The International Society for Optical Engineering, vol. 2545, pp. 108-119, 1995.

[11] N. Dumas, C. Trigona, P. Pons, L. Latorre, and P. Nouet, "Design of smart drivers for electrostatic MEMS switches," Sensors and Actuators A: Physical, vol. 167, no. 2, pp. 422-432, 2011.

[12] R. Lewandowski and P. Wielentejczyk, "Analysis of dynamic characteristics of viscoelastic frame structures," Archive of Applied Mechanics, vol. 90, no. 1, pp. 147-171, 2020.

[13] L. Zhang, X. Zhang, J. Song, and H. Zheng, "Thermo-induced curvature and interlayer shear stress analysis of MEMS double-layer structure," Continuum Mechanics and Thermodynamics, vol. 32, no. 4, pp. 1127-1139, 2020.

[14] Y. Kessler, A. Liberzon, and S. Krylov, "Flow velocity gradient sensing using a single curved bistable microbeam," Journal of Microelectromechanical Systems, vol. 99, pp. 1-6, 2020.

[15] L. Ruzziconi, N. Jaber, L. Kosuru, M. L. Bellaredj, and M. I. Younis, "Internal resonance in the higher-order modes of a MEMS beam: experiments and global analysis," Nonlinear Dynamics, vol. 103, no. 3, pp. 2197-2226, 2021.

[16] M. Mousavi, M. M. Zand, and S. Siahpour, "Effect of added mass distribution on the dynamic PI and frequency shifting in MEMS and NEMS biosensors," Microsystem Technologies, vol. 27, no. 5, pp. 1-10, 2020.

[17] M. Rezaee and N. Sharafkhani, "Nonlinear dynamic analysis of an electrostatically actuated cylindrical micro-beam subjected to cross fluid flow," International Journal of Applied Mechanics, vol. 11, no. 6, pp. 745-755, 2019.

[18] D. M. Lancaster, C. H. Allen, K. Jersey, and T. Lancaster, "Electrostatic guiding of the methylidyne radical at cryogenic temperatures," The European Physical Journal D, vol. 74, no. 6, 2020.

[19] R. K. Godara, A. K. Sharma, N. Joshi, and M. Joglekar, "A novel capacitive mass sensor using an open-loop controlled microcantilever," Microsystem Technologies, vol. 26, no. 4, 2020. 